\title{
Haptic Stylus with Inertial and Vibro-Tactile Feedback
}

\author{
Atakan Arasan, Cagatay Basdogan, T. Metin Sezgin
}

Koc University

\begin{abstract}
In this paper, we introduce a novel stylus capable of displaying two haptic effects to the user. The first effect is a tactile flow effect up and down along the pen, and the other is a rotation effect about the long axis of the pen. The flow effect is based on the haptic illusion of "apparent tactile motion", while the rotation effect comes from the reaction torque created by an electric motor placed along the stylus shaft. The stylus is embedded with two vibration actuators at the ends, and a DC motor with a rotating balanced mass in the middle. We show that, it is possible to create flow and rotation effects on the stylus by driving the actuators on the stylus. Furthermore, we show that the timing and the actuation patterns of the vibration actuators and DC motor on the stylus significantly affect the discernibility of the synthesized perceptions; hence these parameters should be selected carefully. Two psychophysical experiments, each performed with 10 subjects, shed light on the discernability of the two haptic effects as a function of various actuation parameters. Our results show that, with carefully selected parameters, the subjects can successfully identify the flow of motion and the direction of rotation with high accuracies.
\end{abstract}

KEYwORDS: haptics, vibrotactile, inertia, torque perception, tactile illusions.

IndeX Terms: [Communication Hardware, interfaces and storage]: Tactile and hand-based interfaces-Haptic devices; ¡Human computer interaction]: Interaction devices-Haptic devices; [Computer graphics]: Graphic systems and interfacesPerception;

\section{INTRODUCTION}

The pen is an appropriate input device for intuitive and direct interaction. Using a stylus in combination with an electronic display closely resembles the familiar pen and paper arrangement. Many interaction tasks, such as drawing, writing and manipulation can be easily done with a pen-based interface. Hence, today, most tablet PCs and mobile devices support stylus to interact with graphical interfaces in a convenient way. Such pen-based interfaces enable a user to interact with the data and the objects displayed on the screen, but do not provide the user with haptic feedback during selection or manipulation. Previous studies have already shown that using haptic feedback for conveying information over the tactile channel improves manipulability and enriches the interaction experience [1]. In particular, human fingertips have the most discriminating tactile sensors [2], and these sensors enable the user to experience vibration stimuli in various forms. Hence, we developed a haptic stylus that can

College of Engineering, Koc University, Istanbul, Turkey, 34450. E-mail: aarasan, cbasdogan, mtsezgin@ku.edu.tr

IEEE World Haptics Conference 2013

14-18 April, Daejeon, Korea

978-1-4799-0088-6/13/\$31.00 @2013 IEEE display haptic effects to a user through the tactile channel.

Our haptic stylus includes two vibration motors, one positioned close to the tip, and the other close to the end of the stylus. Also, a DC motor is located inside the body of the pen, positioned directly underneath the fingers as they hold the pen (Figure 1). In an effort to display tactile feedback through the stylus, we made use of contact points as multiple fingertips and the skin area between proximal phalanx of thumb and index finger.

Using the proposed stylus, we display two illusionary haptic effects to a user: one is a flow effect along the long axis of the pen and the other is a rotation effect, about the long axis of the pen. The former is based on a sensory illusion, known as apparent tactile motion [3], which is generated by the use of two vibration motors. The idea behind apparent tactile motion is to vibrate the body of the stylus by the motors with an appropriate combination of stimulus duration and inter-stimulus onset interval (ISOI), which creates a tactile flow effect rather than a single stimulation or a set of sequential stimulations.

The other haptic effect displayed through the stylus is clockwise or counterclockwise rotation about the long axis of the stylus. To create this haptic effect, we actuate a high-torque DC motor placed at the center of the stylus for a brief period of time. When a voltage pulse is sent to the motor (i.e. the on-time), the user holding the casing perceives a torque feedback about the intended rotation direction until the motor reaches to its maximum rotational speed for that voltage. During the off-time, a reaction torque in the opposite direction is displayed to the user by the motor due to its inertia. When the motor is turned on and off for carefully tuned durations, the stylus responds in a way that is perceived by the user as a clockwise or a counter-clockwise rotation about the long axis of the pen.

We determined the effective values of tactile stimulus for creating both of these illusionary effects via two separate psychophysical experiments, each performed with 10 subjects. In the first experiment, we investigated the control parameters for apparent tactile motion that allow "single stationary" (i.e. single tap), "discrete" (i.e. double tap) and "continuous" (i.e. flow of motion) vibrotactile stimuli. For the continuous case, we evaluated the effects of changing the stimulus duration and ISOI on the perceived direction of flow along the stylus. In the second experiment, we investigated the effect of on-time and off-time on the perceived direction of rotation for three consecutive torque pulses. In addition, further insight on rotational tactile effect was sought by attaching a force/torque sensor to the pen and conducting a torque test. We investigated the relation between the torque applied to the pen and the perceived direction of rotation.

\section{RELATED WORK}

In haptics literature, only a few studies are available on penbased interfaces displaying tactile feedback to a user. Most of the earlier studies focused on displaying tactile effects. Lee et al. developed a stylus with a solenoid actuator at the tip to display interaction forces to a user along the longitudinal axis of the stylus [4]. Kyung and Jun-Seok proposed a stylus with an embedded pin array to display tactile patterns at the fingertip of a user [5]. Also, a vibration motor was attached to the body of the stylus to generate a sense of contact with a surface. Kamuro et al. 
developed an ungrounded kinesthetic display which is capable of bending about a pivot point to facilitate three-dimensional interaction [13]. Withana et al. proposed a haptic stylus which changes its effective length when the stylus pushed into a touch screen [14].

Except Kamura et al. [13], all other designs contain single actuator and they lack the ability to render the wide variety of effects that our system is capable of. The richness of haptic effects displayed through a stylus can be increased by using multipleactuators instead of a single one. Using multiple-actuators, wide range of haptic effects can be generated by altering not only the frequency, intensity, and duration of a single stimulus, but the location, spacing, and direction of the actuators, as well as the synchronization between them. For example, by altering the stimulus duration and ISOI of the actuators, it is possible to create a tactile illusion of continuous motion, also known as apparent tactile motion [3]. Similarly, if the intensities of the stimuli are altered, one can create a "Phantom" sensation, which is perceived as an illusionary vibrating actuator located between the real actuators [9]. These tactile illusions generated by multiple actuators have been used in different application domains including wearable computing [12], gaming [6], mobile devices [10], and, automotive technology [11].

In our study, we adopt the concept of apparent tactile motion to generate and display a flow effect along the long axis of our stylus by means of two vibration motors. Additionally, a rotational direction cue about the long axis of the stylus is provided to the user via a DC motor. Rotational sliding displays and inertial systems have been suggested for displaying directional haptic cues. In [7], a set of experiments were conducted to understand the human tactile discrimination thresholds for perceived rotation rate at the fingertip. In their setup, the index finger of the user was in contact with a rotating disk. The friction between the fingertip and the disk surface generated a haptic cue for the direction of rotation. Nakamura et al. developed a portable and mobile cube to display rotational direction cues to the palm of a user holding the cube [8]. The directional cues were generated by controlling the voltage signals to the three orthogonally positioned motors inside the cube. Amemiya et al. implemented directional torque feedback in mobile devices through abrupt start and stop of a flywheel [15]. In [16], using gyroscopic effect, directional torque feedback was generated by a flywheel surrounded by two-axis actuated gimbal.

The remainder of the paper is organized as follows: In Section 3 , we present the design of the hardware and software parts of our haptic stylus. In addition, the methods used for creating and displaying a) the flow and b) rotational haptic effects are given in the same section. In Section 4, the procedures and the results of the two psychophysical experiments are presented. Finally, Section 5 presents a discussion of the results and future work.
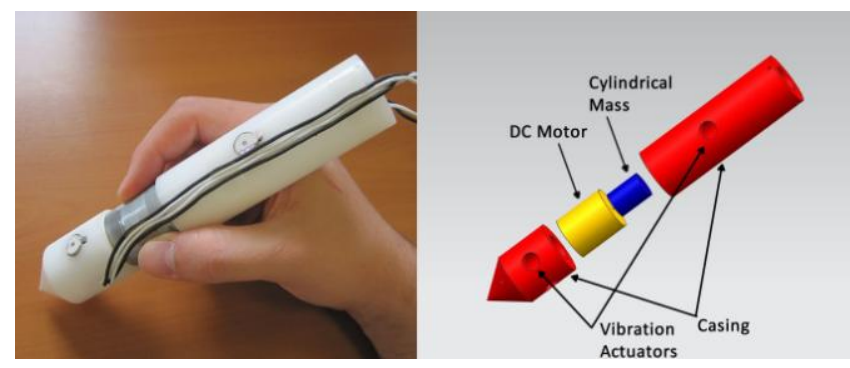

Figure 1. Our stylus and its components

\subsection{Design of the Haptic Stylus}

Our haptic stylus is a simple and a low-cost apparatus designed for displaying tactile feedback to enhance interaction with graphical interfaces. Our stylus consists of a cylindrical casing and physical actuators integrated into the casing. The choice of the physical actuators is critical for the effectiveness and expressiveness of the tactile feedback displayed to the user.

To create a flow effect via vibrotactile stimulus, linear resonant actuators (LRAs), eccentric rotating masses (ERMs), or piezo actuators could be utilized. We have chosen coin type ERM actuators over LRA and piezo actuators. Coin type ERM actuators are compact and lightweight, and have a larger bandwidth than coin type LRA actuators. Moreover, coin LRA actuators vibrate in the axial direction (i.e. normal to the coin surface) whereas coin type ERM actuators produce radial vibrations. Hence, the vibration signals are better transmitted to the body of the stylus through the use of ERMs. The piezo actuators were not preferred for the design since their power consumption is typically higher than vibration motors. Two coin type ERM actuators (model C1026B200F; Kysan Electronics Inc.) were placed close to the tip and the end of the stylus (Figure 1). To improve the performance of the actuators, a haptic motor driver (model DRV8601, Texas Instruments) was used. The driver was optimized to drive ERMs and LRAs with a fast turn-on time $(0.1 \mathrm{~ms})$ using only a singleended pulse width modulation (PWM) input signal.

To create rotational direction effect, a brushed DC Motor with a high rated torque was used. This motor was placed around the center of the stylus close to the tip (Figure 1). Short voltage pulses sent to the motor produce a torque for a short duration. To amplify the effect of torque, a cylindrical balanced mass was attached to the output shaft of the motor. Besides the inertia of the armature, the additional inertia coming from the cylindrical mass increases the torque transmitted to the user through the casing. A motor driver (model L293D; ST Microelectronics Inc.) was used to

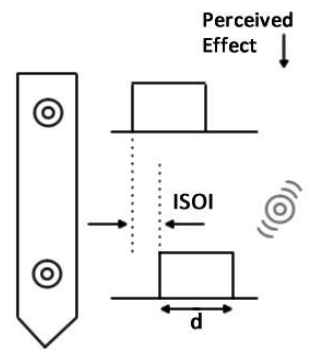

a) Single Stationary

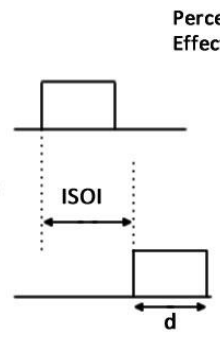

b) Discrete

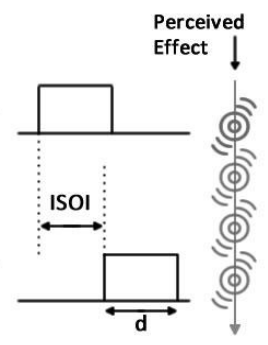

c) Continuous
Figure 2. For a constant stimulus duration, effect of ISOI on the tactile flow effect displayed through the stylus

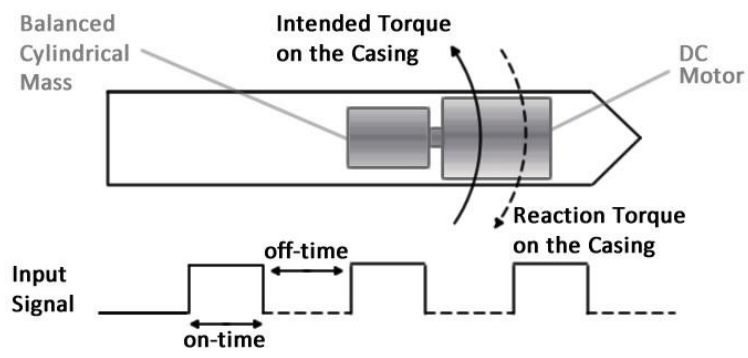

Figure 3. Rotational Direction Effect: during on-time, motor generates intended torque on the casing and during off-time, reaction torque is generated on the casing 
control the motor. This driver is specifically designed to control high torque DC and stepper motors. The communication between the drivers and the host PC was achieved by a microcontroller (model ATTINY 2313; Atmel Inc.). The microcontroller receives the commands from the host PC via UART (Universal Asynchronous Receiver/Transmitter) at 38400 baud rate and generated PWM signals at $125 \mathrm{~Hz}$ for the drivers.

\subsection{Haptic Effects}

The principles underlying the synthesis of the two effects that our stylus can render are based on the so-called flow effect and the rotational direction effect.

\subsubsection{Flow Effect}

When two vibration motors placed in close proximity on the skin are actuated with overlapping actuation times, they are not perceived as two separate actuators, but as a virtual actuator moving between them (Figure 2). This sensory illusion is known as apparent tactile motion and has been studied since the early 1900s [6]. This illusion is achieved by adjusting the duration of vibrotactile stimulus and the inter-stimulus onset interval (ISOI), i.e. the duration between the onsets of the actuators separated. While this illusion has been demonstrated on human forearm and back [6], to our knowledge, it has not been implemented on a penbased interface. Moreover, earlier works only considered the cases where the actuators are in full contact with the skin, but we show that this illusion is still very effective when there is no direct contact with the actuators as in our case.

\subsubsection{Rotational Direction Effect}

Torque generated by a motor is proportional to the derivative of angular velocity as well as the inertia of the armature and the load on the output shaft, which is formulated as follows

$$
\tau=\left(\mathrm{J}_{\text {Armature }}+\mathrm{J}_{\text {Load }}\right) *(d \sigma / d t)
$$

In our design, we attach a cylindrical balanced mass to the output shaft of the actuator to increase the total moment of inertia, hence the torque displayed to the user through the DC motor. The additional mass is an alternative solution to increase the torque rather than replacing the motor with a larger one having a higher rated torque. In this way, we keep the stylus compact in size.

When a voltage pulse is sent to the motor, the rotational speed reaches to a maximum value, $\omega_{\max }$, for that voltage during the ontime (Figure 3). During the off-time, a reaction torque $\tau_{\mathrm{r}}$ is generated in direction opposite to the intended direction of torque $\tau$. Even a reaction torque $\tau_{\mathrm{r}}$ exists, the user still perceives the direction of the intended rotation successfully if the on- and offtime of the pulses are selected carefully. In our preliminary experiments, we observed that three pulses are sufficient to effectively convey the direction of the rotation.

\section{EXPERIMENTAL RESULTS}

We conducted two separate experiments. The goal of the experiments was to determine the effective values of stimulus parameters for displaying the proposed illusions through the stylus. Two separate groups of 10 subjects participated in each experiment. The average age of the subjects in the first and the
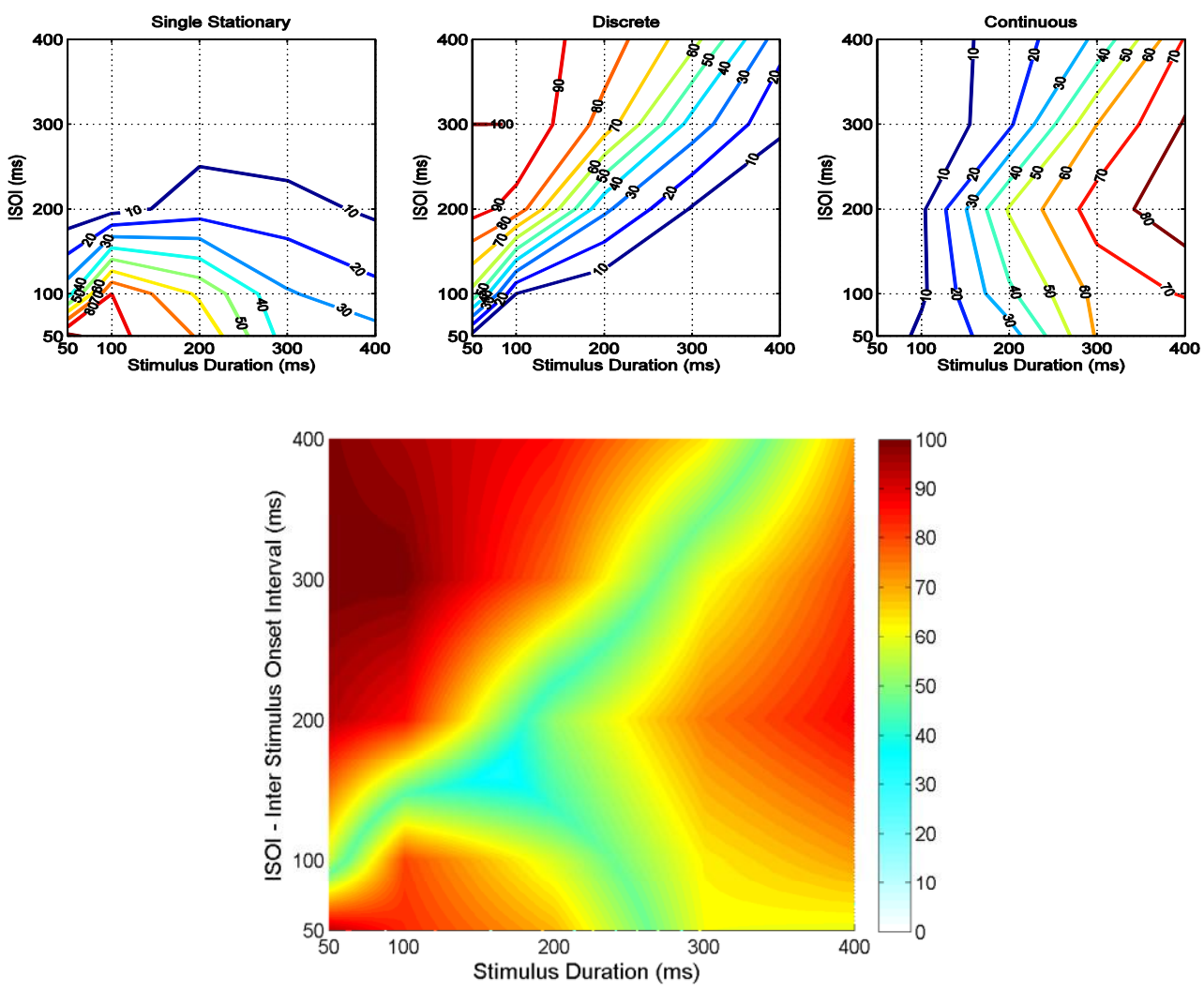

Figure 4. Tactile Flow Effect: The contour graphs on the top row display the percentage of the votes given by users to each rating class (single stationary, discrete, and continuous) for combinations of various ISOI and stimulus duration values. The bottom graph combines these plots and shows the rating of the most dominant effect at each point in terms of percentages. Note how the valleys in the combined plot clearly divide the space into three distinct regions corresponding to distinct effects. 

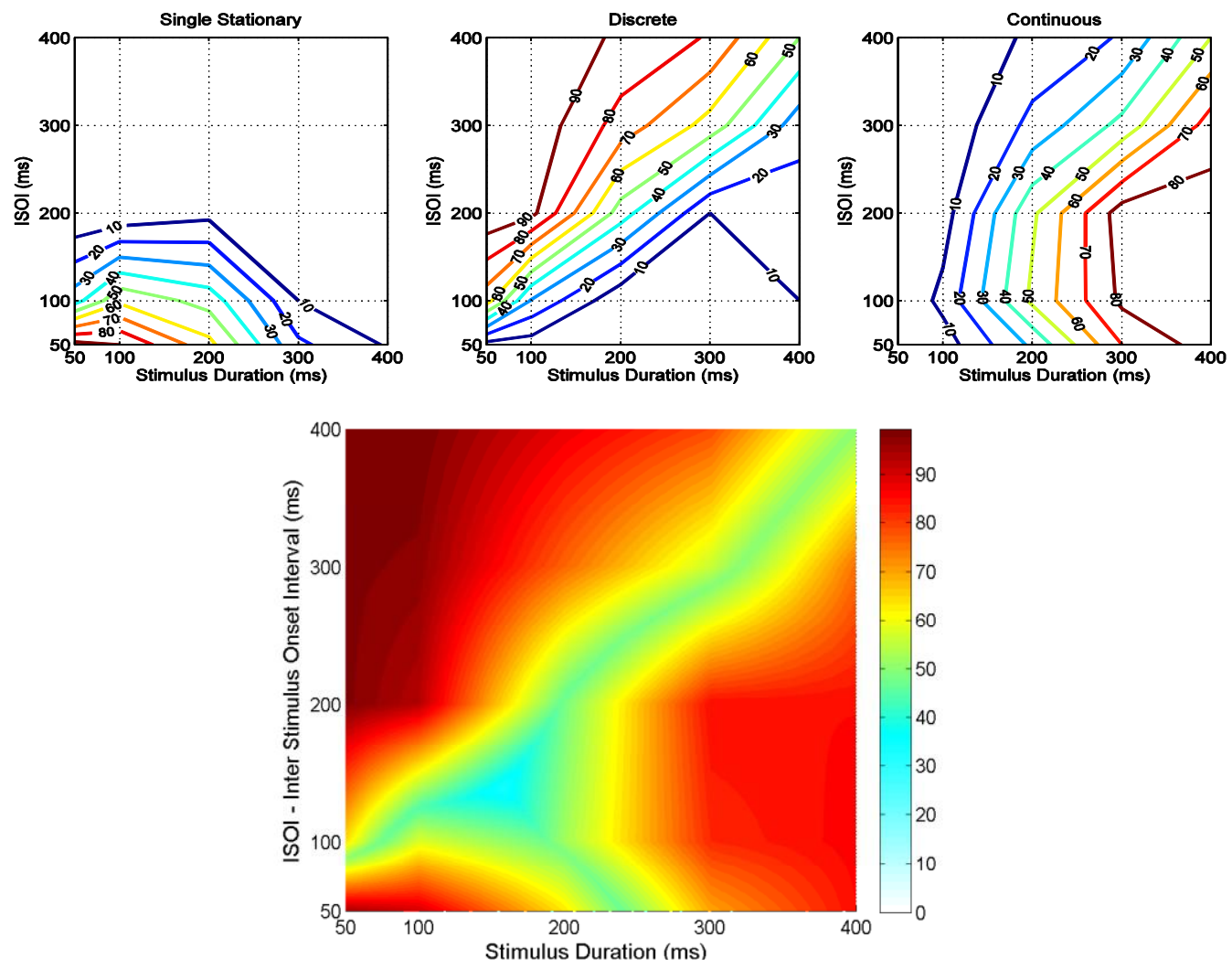

Figure 5. Tactile Flow Effect: The contour graphs on the top row display the percentage of the votes given by users to each rating class (single stationary, discrete, and continuous) for combinations of various ISOI and stimulus duration values. The bottom graph combines these plots and shows the rating of the most dominant effect at each point in terms of percentages. Note how the valleys in the combined plot clearly divide the space into three distinct regions corresponding to distinct effects.

second experiments were $24 \pm 2$ and $24 \pm 3$, respectively. The participants did not have any known sensory impairments and had not participated in a user study related to haptics before. They were all right handed. Hence, they used their right hand to hold the stylus and left hand to enter their responses by pressing the keys on the keyboard. During the experiments, the subjects sat comfortably on a chair facing towards the computer screen displaying the experimental protocol and put on headphones that played "sounds of nature" to block auditory cues. In the second experiment, a visual barrier was put in place to prevent the subjects from seeing their hand and the stylus.

\subsection{Experiment 1}

In the first experiment, the goal was to determine the effective values of stimulus duration and ISOI for displaying the tactile flow effect. Five different values, 50, 100, 200, 300, and $400 \mathrm{~ms}$, were used for stimulus duration and ISOI. Two flow directions along the long axis of the stylus were investigated: from the tip of the stylus to the end of the stylus and vice versa. The experiment consisted of a total of 500 trials: 50 trials ( 5 stimulus durations x 5 ISOIs $\mathrm{x} 2$ directions) with 10 repetitions per trial. The subjects completed the experiment in two sessions in the same day: one session (250 trials) for each direction. Each session took no more than 20-25 minutes and there was a break of at least 3 hours between the sessions. In each trial, the subjects were asked to characterize the tactile stimulus displayed through the vibration motors as "single stationary", "discrete", or "continuous". The trials in each session were randomized to eliminate any bias.
Before starting each session, the subjects were presented a training session including all possible combinations of the parameters $(25$ trails $=5 \times 5)$.

\subsection{Experiment 2}

In the second experiment, the goal was to determine effective values of on- and off-time durations of the 3 pulses sent to the DC motor for displaying rotational direction effect. Six different values, $25,75,175,275,375$, and $575 \mathrm{~ms}$, were used for on-and off time durations of the motor for clockwise and counterclockwise rotations about the long axis of the stylus. There were a total of 720 trials in this experiment: 72 trials (6 on-time durations $\mathrm{x} 6$ off-time durations $\mathrm{x} 2$ directions) with 10 repetitions of each trial. The subjects completed the experiment in three sessions in the same day. Each session took no more than 20-25 minutes and there was a break of at least 2 hours between the sessions. In each trial, the subjects were asked to differentiate if the torque displayed through the DC motor as "clockwise", or "counterclockwise" with respect to the long axis of the stylus. The trials in each session were randomized to eliminate any bias. Before starting each session, the subjects were presented a training session including all possible combinations of the parameters (36 trails $=6 \times 6$ ).

\subsection{Results}

Figure 4 and Figure 5 are concise graphs showing the effect of stimulus duration and ISOI on the perception of the subjects in the first experiment. Both figures illustrate that three regions are 
clearly separated from each other: the bottom left region representing the "single stationary", the top left region representing the "discrete", and the middle right region representing the "continuous" stimuli, as perceived by the subjects.

The response of the subjects was analyzed by a Three-Way Repeated Measures Analysis of Variance (ANOVA). We investigated the effects of stimulus duration, ISOI, and the direction of tactile flow on the perception of the subjects. A significance level of $\alpha=0.05$ was used throughout the analysis of the results. ANOVA analysis suggested that duration of stimulation is a significant factor $[\mathrm{F}(4,36)=102.65, \mathrm{p}<0.05]$. Paired t-tests showed that the accuracy of tactile flow perception is higher as stimulus duration increases $(p<0.05)$ (Figure $7 a)$. The ISOI was also a significant factor affecting the perception of the subjects $[F(4,36)=7.18, p<0.05]$. Paired t-tests showed that the stimulus was perceived as "continuous" as ISOI was between 50 and $200 \mathrm{~ms}(\mathrm{p}<0.05)$. For example, at the stimulus duration of 300 $\mathrm{ms}$, the percentages of the "continuous" response perceived by the subjects for ISOI 50,100, and $200 \mathrm{~ms}$ here significantly high that those of ISOI 300 and $400 \mathrm{~ms}$ (Figure 7b). ANOVA analysis

a) Clockwise

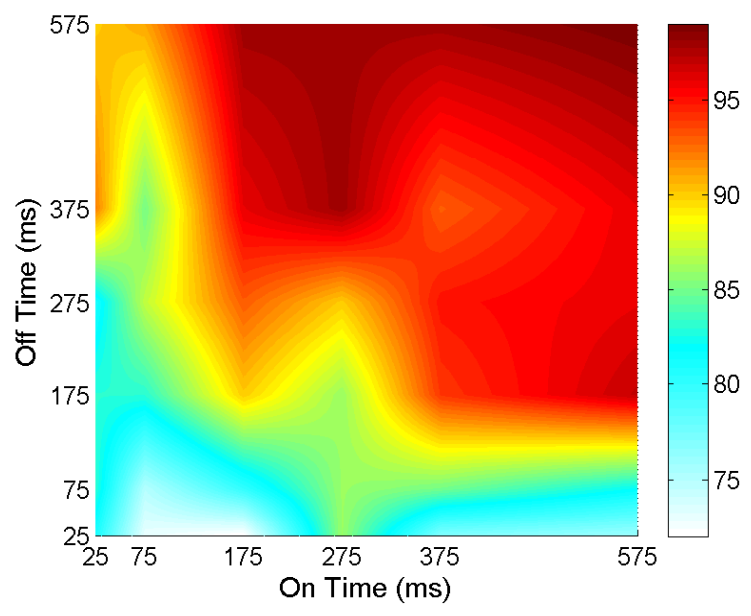

b) Counter-Clockwise

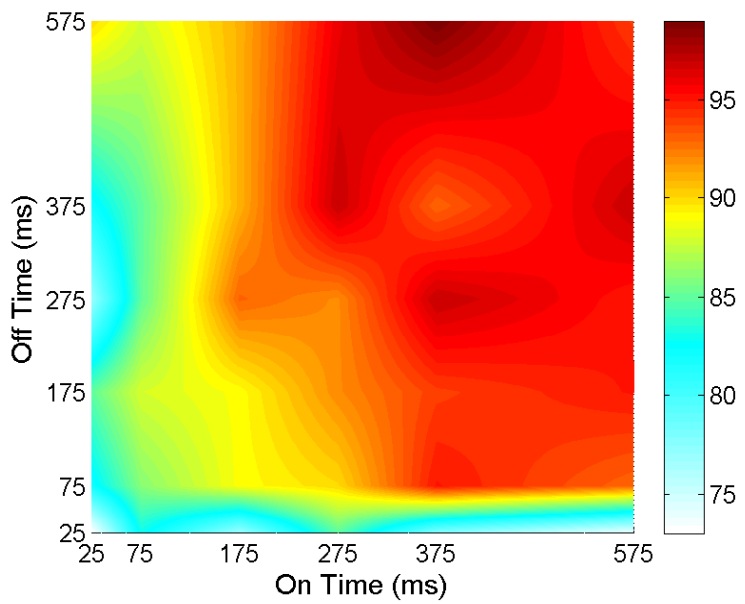

Figure 6. Rotational Direction Effect. The tactile effect is given in a) clockwise b) counter-clockwise directions on the stylus. The color bars next to the plots indicate the accuracy of perceived direction. showed that the direction of flow was not a significant factor on the decision of the subjects $[\mathrm{F}(1,9)=0.03, \mathrm{p}=0.86]$. In other words, the subjects correctly judged the type of stimulus independent of the direction of flow. This is also evident in Figure 4 and Figure 5 since both plots are quite similar to each other.

Figure 6 is a concise graph showing the effect of on and offtime durations on the perception of the subjects in the second experiment. In each plot, red colored region represents the parameter space in which the direction of rotation was perceived successfully by the subjects. The responses of the subjects were also analyzed by a Three-Way Repeated Measures Analysis of Variance (ANOVA). The analysis was utilized to investigate the effects of on and off-time durations on the perception of the subjects. A significance level of $\alpha=0.05$ was used throughout the analysis. ANOVA results suggested that durations of on and offtimes significantly influence the decision of the subjects about the direction of rotation $[\mathrm{F}(5,45)=4.49, \mathrm{p}<0.05,[\mathrm{~F}(5,45)=4.49$, $\mathrm{p}<0.05]$, respectively]. The results showed that the subjects have identified the direction of rotation more successfully at the higher on- and off-time durations (Figure $7 \mathrm{c}, \mathrm{d}$ ). The results of ANOVA showed that the perception of rotational direction was not significantly affected by the selected direction $[F(1,9)=0.03$, $\mathrm{p}=0.86]$. Hence, the perception of direction only depends on the on and off time durations. a)

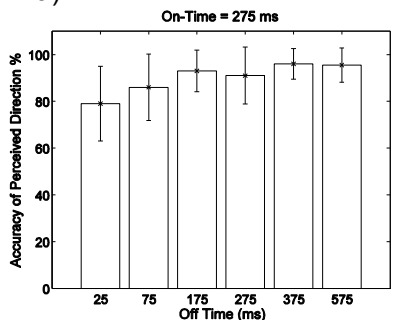

c)

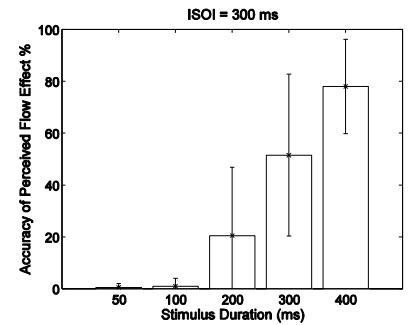

b)

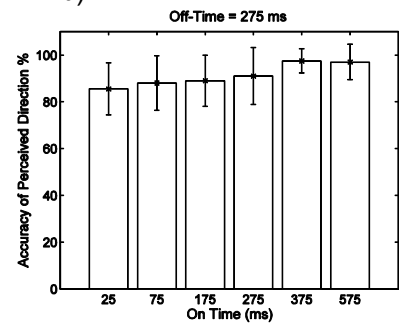

d)

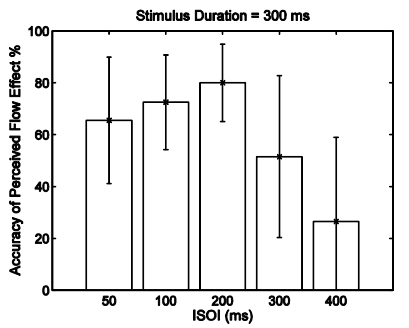

Figure 7. Accuracy of perception with standard deviation for flow of motion a) with constant ISOI b) with constant stimulus duration and rotational direction c) with constant on-time d) with constant off time

\section{DISCUSSION}

The results of first experiment demonstrate that the sensation of tactile flow can be reliably created on a stylus by using two vibration actuators. Among the three parameters, stimulus duration and ISOI significantly alter the perceptual characteristics of the tactile flow but the direction of flow has no effect. Higher duration and lower ISOI values increase the perception of a "continuous" flow as shown in Figure 4 and Figure 5. The effective values of parameters for perceiving "single stationary", "discrete", and "continuous" stimuli are different than those reported in previous studies on apparent tactile motion performed on human forearm and the back [6]. This can be explained by the 
fact that human hand and fingertips are more sensitive to vibrations than human forearm and back.

The results of the second experiment show that the rotational haptic cues about the long axis of the stylus can be successfully perceived by the subjects. Our results demonstrate that the subjects are more successful in perceiving the intended rotational direction at higher on- and off-time durations of the motor. To better understand the results of the second experiment, we measured the torque response of the stylus using a force/torque sensor (model Nano 17, ATI Inc.). For this purpose, the force/torque sensor was connected to a rigid support and the end of the stylus was attached to the sensor. The DC motor at the center of the stylus was actuated with the same on-and off-time durations used in the second experiment. The output torque about the long axis of the stylus was recorded by the sensor at a rate of 1 $\mathrm{kHz}$ with a resolution of $1 / 64 \mathrm{Nmm}$. A Gaussian low pass filter was used to clean the noise from the measured torque response.

Figure 8 shows the torque output of the stylus for a constant ontime duration of $275 \mathrm{~ms}$ and three different off time durations of 75,175 , and $375 \mathrm{~ms}$. Note that two voltage pulses sent to the DC motor, creating two torque pulses on the stylus. Recall that the perception of rotational direction was highest for the off-time 375 $\mathrm{ms}$ followed by $175 \mathrm{~ms}$ and $75 \mathrm{~ms}$ respectively. We observe that longer off-time values allow the torque response to drop back to the equilibrium state (i.e. $\tau=0$ ) by the time the motor is actuated for a second time. Also as shown Figure 8, the magnitude of the second pulse is higher when the off-time duration is longer. As a result, the average magnitude of the torque transmitted to the user is higher about the intended direction.

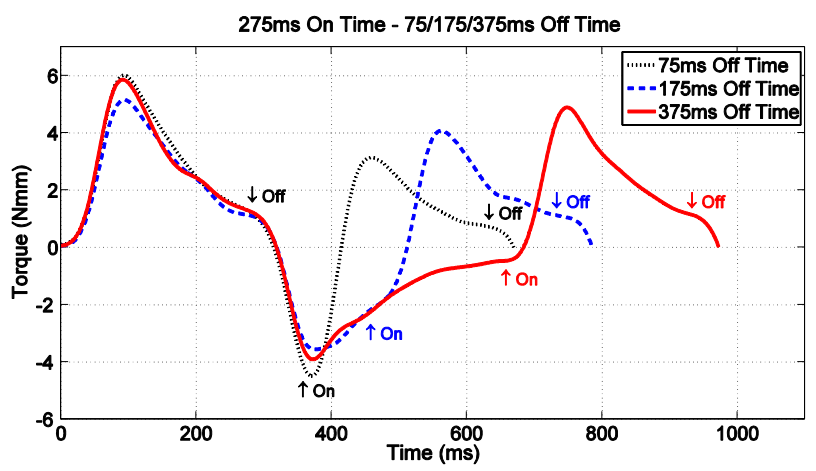

Figure 8. The system torque for various on- and off-time values. Recall Experiment 2.

\section{CONCLUSION}

In this paper, we reported two approaches for creating the sensation of flow and rotation on a pen-based interface, and we examined the parameter spaces of these illusionary haptic effects. We showed that apparent tactile motion for a stylus (a hand held device) differs from those reported by studies designed for different parts of the body such as the back and the forearm. We also show with a psychophysical experiment and a torque test that a rotational haptic illusion can be given by consecutive sudden starts and stops on the pen-based interfaces.

Future work will include embedding more vibrotactile illusions on the pen-based interface such as the phantom illusion. More parameters will be tested for rotational torque feedback and we also plan on designing a more compact stylus design expected to be presented. Also an ergonomic design can be investigated to increase the perception of the proposed haptic illusions in this paper.

\section{REFERENCES}

[1] S. Choi and H. Z. Tan., "Toward realistic haptic rendering of surface textures," ACM SIGGRAPH 2005 Courses (SIGGRAPH '05), vol. 24, no. 2, pp. 40-47, 2005.

[2] R. S. Johansson, and Å. B. Vallbo. "Tactile sensibility in the human hand: relative and absolute densities of four types of mechanoreceptive units in glabrous skin," The Journal of physiology vol. 286, pp. 283-300, 1979.

[3] C. Sherrick, and R. Rogers. "Apparent haptic movement," Attention, Perception, \& Psychophysics, vol. 1, pp. 175-180, 1966.

[4] J. C. Lee, P. H. Dietz, D. Leigh, W. S. Yerazunis, and S. E. Hudson. "Haptic pen: a tactile feedback stylus for touch screens," In Proceedings of the 17th annual ACM symposium on User interface software and technology (UIST '04), pp. 291-294, 2004.

[5] K. Kyung, and J. Park. "Ubi-Pen: Development of a compact tactile display module and its application to a haptic stylus," Proceedings of World Haptics, pp. 109-114, 2007.

[6] A. Israr, and I. Poupyrev. "Tactile brush: Drawing on skin with a tactile grid display," Proceedings of the 2011 annual conference on Human factors in computing systems. ACM, pp. 2019-2028, 2011.

[7] W. R. Provancher, B. D. Erickson, F. Barbagli, H. Z. Tan. "Tactile perception of rotational sliding, Proceedings of World Haptics, pp.127-132, 2007.

[8] M. Sakai, Y. Fukui, and N. Nakamura. "Effective output patterns for torque display 'GyroCube'," Online Proceeding of the 13th International Conference on Artificial Reality and Telexistence, vol. 13, pp. 160-165, 2003.

[9] D. S. Alles. "Information transmission by phantom sensations," Transactions on Man-Machine Systems, vol. 11, pp. 8591, 1970.

[10] J. Kim and K. Kim. "Traveling vibrotactile wave-a new vibrotactile rendering method for mobile devices," IEEE Transactions on Consumer Electronics, vol. 55, pp. 1032-1038, 2009.

[11] E. S. Ege, F. Cetin, and C. Basdogan. "Vibrotactile feedback in steering wheel reduces navigation errors during GPS-guided car driving," Proceedings of World Haptics Conference, pp. 345-348, 2011.

[12] H. Z. Tan and A. Pentland. "Tactual displays for wearable computing," Personal and Ubiquitous Computing, vol. 1, pp. 225230, 1997.

[13] S. Kamuro, K. Minamizawa, and S. Tachi. "An ungrounded penshaped kinesthetic display: Device construction and Applications," Proceedings of World Haptics Conference, pp. 557-562, 2011.

[14] A. Withana, M. Kondo, Y. Makino, G. Kakehi, M. Sugimoto, and M. Inami. "ImpAct: Immersive haptic stylus to enable direct touch and manipulation for surface computing," ACM Computers in Entertainment, vol. 8, no. 9, 2010.

[15] T. Amemiya and H. Gomi. "Directional torque perception with brief, asymmetric net rotation of a flywheel," IEEE Transactions on Haptics, to appear.

[16] K. Winfree, J. Gewirtz, T. Mather, J. Fiene, and K. Kuchenbecker. "A high fidelity ungrounded torque feedback device: The iTorqU 2.0," Proceedings of World Haptics, pp. 261-266, 2009. 